\title{
Identification of Silver Nanoparticle-shaping Tridax procumbens Phytoconstituent by Theoretical Simulation and Experimental Correlation
}

\author{
N. IRFAN AND A. PURATCHIKODY* \\ Drug Discovery and Development (D3) Research Group, Department of Pharmaceutical Technology, Bharathidasan Institute \\ of Technology, Anna University, Tiruchirappalli-620 024, Tamilnadu, India

\begin{abstract}
Irfan and Puratchikody: Identification of Silver Nanoparticle-reducing Phytoconstituent by in silico Simulation
\end{abstract}

\begin{abstract}
In this present work, silver nanoparticles were synthesized, the biological mechanisms involved and interaction routes were explained using density function theory, adsorption site identification and blend binding energy calculations. The results indicated that among the 18 molecules of a methanol fraction of Tridax procumbens only the phytoconstituent, baicalin was accountable for materialization of the silver nanoparticle. Baicalin formed six $\pi$-cationic interactions with a silver ion, which formed the repulsive network between each other atom to rule out the binding of nitrate ion. The HOMO, LUMO and binding energies conformed that baicalin-Ag complex was stable. These fingerprint simulation studies pointed out the easy way for researchers in green nanotechnology for identifying nanoparticle-forming phytoconstituents from the plant extracts in future.
\end{abstract}

Key words: Tridax procumbens extract, silver nanoparticles, density function theory, adsorption locator, Blends binding energy, nano interaction

Nanotechnology is an emerging interdisciplinary technology involving biology, medicine, chemistry and material sciences ${ }^{[1]}$. Over the past decades, nanoengineered particles have provoked much curiosity due to their distinct physicochemical and biological properties ${ }^{[2]}$. Nanoparticles (NPs) considered as structure slabs and the key to the next generation technology in many fields ${ }^{[3]}$. Especially, silver nanoparticles (AgNPs) are one of the supremely energetic and captivating nanomaterials among several metallic NPs frequently used in the biomedical zone ${ }^{[4]}$. Although, several noble metals used for various purposes, in that AgNPs engrossed applications in cancer diagnosis and therapy. There are several physical and chemical methods for the synthesis of silver nanomaterials. However, due to the low yield and use of toxic solvents hazardous to the environment the medical applications of synthesized NPs are getting limited ${ }^{[5,6]}$.

The growing need to eliminate the environmental risk substances, as green chemistry ideologies labeled the synthesis of NPs using nano green field has received increasing attention in the last decade ${ }^{[7]}$. Nano green is merging the green chemistry and green engineering with nanotechnology. This environment-friendly approach has much more prominence due to its economic advantage, biocompatibility and viability in the long run, principally in the medical field ${ }^{[8,9]}$. Naturally grown plants, which are a dynamic source of phytochemicals, serve as a reservoir for the production of metallic NPS. Research indicated that nucleation and growth of NPs occur inside the plants ${ }^{[10]}$. This fact gave rise to the possibility to use plant phytoconstituents for nanoparticle synthesis, which could be advantageous over other biological processes. Bioengineering of metal NPs based on the assortment of reducing agents, solvent medium and stabilizing agents. Generally, plant extract contains both a reducing and stabilizing agents, constituents, which are responsible for the molecularlevel modifications of their metabolic weaponry,

This is an open access article distributed under the terms of the Creative Commons Attribution-NonCommercial-ShareAlike 3.0 License, which allows others to remix, tweak, and build upon the work non-commercially, as long as the author is credited and the new creations are licensed under the identical terms

Accepted 10 August 2019

Revised 17 May 2019

Received 04 January 2019

Indian J Pharm Sci 2019;81(5):900-912 
resulting in the synthesis of either metallic or oxide $\mathrm{NPs}^{[11,12]}$.

In the present work, Tridax procumbens leaf extract (microwave irradiation) was used to synthesize AgNPs. T. procumbens is one such multidimensional weed available throughout the region, which used as a supernumerary for many herbs. The phyotochemical examination reported the presence of lipid constituents, sterols, bergenin derivatives and flavonoids ${ }^{[13-15]}$. However, the diverse compositions of $T$. procumbens leaf extracts do not explicitly answer what is responsible for the metal ions reduction. Hence, this work was taken up to identify the AgNP shaping phytoconstituent using novel strategic dynamic simulation approaches such as adsorption locator, HOMO/LUMO energy, density function theory (DFT) and blend binding analysis. The reaction and binding energies of phytoconstituents on silver metal surfaces were investigated using molecular level interaction. Finally, the theoretical results correlated with experimental values to conclude the nano-sized silver particle shaping phytoconstituent of $T$. procumbens. Moreover, the available spectroscopic techniques have provided only insufficient and indirect information of binding events. Therefore, for understanding selective orbital binding, effects of chemical functionalities and resulting non-covalent interactions of phytoconstituent were explained in this work.

\section{MATERIALS AND METHODS}

Reagents used in this study were all the first grade and HPLC grade. Microwave-assisted extraction (MAE) was performed in commercial open type Catalysis-4RI microwave synthesizer. It can emit $140-700 \mathrm{~W}$ in $15 \mathrm{~W}$ microwave increments with beam reflector for perfect distribution of microwaves. The flexible dipping type temperature probe was used to calculate the temperature. The solvent extracts stored in amber colored pyrogenfree Tarsons click lock microcentrifuge tubes for further factor analysis. Whatman filter papers (20$25 \mu \mathrm{m}$ pore size) and a rotary vacuum evaporator (IKA RV 10) were used to separate the phytoconstituents from the final solvent extract.

\section{T. procumbens plant material:}

One to two inches extended, pinnate, arrow-headed, oblong to ovate, opposite, acute apexes, and coarsely serrate marginal leaves of $T$. procumbens collected from Tiruchirappalli region (longitude 78.74545, latitude 10.65774) in August 2016. The collected plant material was identified as Tridax L. genus, T. procumbens species, cited pl. 900. 1753 and Asteraceae family, in the Department of Botany, St. Joseph's College, Tiruchirappalli, Tamilnadu, India. The freshly collected leaves were cleaned with doubledistilled (DD) water to remove dust and other external matters for succeeding use.

\section{Microwave-assisted extraction (MAE):}

Extraction of phytoconstituents from the leaves of T. procumbens was performed in the MAE unit. Fresh plant leaves $(100 \mathrm{~g})$ was kept in a flask of 11 capacity along with $300 \mathrm{ml}$ of DD water. Upon application of 8 (425 watts, $80 \%$ ) level microwave irradiation and the temperate set at $70^{\circ}$ gave better yield. The water molecules residing in the leaf cells rapidly realigned with the electromagnetic field ${ }^{[16]}$. The excited molecules generate a high amount of heat and are vaporized. The increased vapour content inside the cells led to an escalation in internal pressure toward the cell wall, subsequently leading to the rupture of the cell walls. Further, the solvent extract was concentrated and desiccated using a rotary vacuum evaporator.

\section{Fractionation of aqueous extract:}

T. porcumbens aqueous extract was fractionated using an already reported method ${ }^{[17]}$. Dried aqueous extract $10 \mathrm{~g}$ was initially fractionated with $50 \mathrm{ml}$ of the of methanol in a separatory funnel. The solution was shaken vigorously for $15 \mathrm{~min}$, and then the methanol solution was filtered out. All the $50 \mathrm{ml}$ fractions were concentrated to $5 \mathrm{ml}$ and subjected to gas chromatography-mass spectrometry (GC-MS) for the phytochemical analysis.

\section{GC-MS analysis of fractions:}

GC-MS analysis was performed to identify the phytoconstituents present in the methanol fraction of the dried aqueous extract on autosampler attached GC-MS-5975C Agilent system. Column Elite-1 fused silica capillary column $(30 \times 0.25 \mathrm{~mm} \mathrm{ID} \times$ IEM df $)$, functioning in electron impact mode at $70 \mathrm{eV}$; carrier gas helium (99.999\%) was applied at a constant flow of $1.51 \mathrm{ml} / \mathrm{min}$. One microliter injection was delivered with 10:1 split ratio, injector temperature $250^{\circ}$ and ionsource temperature of $230^{\circ}$. The oven temperature was automated from $70^{\circ}-2 \mathrm{~min}$ isothermal, with an increase of $100^{\circ} / \mathrm{min}$, to $300^{\circ} / \mathrm{min}$, ending with $300^{\circ}-9 \mathrm{~min}$ isothermal value. Mass spectra with a scan mass range 40-1000 $\mathrm{m} / \mathrm{z}, 5 \mathrm{~min}$ solvent cut time were recorded. A scan of MS start time is $5 \mathrm{~min}$ to end time to be $35 \mathrm{~min}$. 
National Institute Standard and Technology (NIST) database was used to interpret the mass spectrum of methanol fraction.

\section{Synthesis of NPs:}

Concentrated methanol fraction of T. procumbens was added to the aqueous solution of $1 \mathrm{mM}$ silver nitrate (AgNO3, MW 169.87, Sigma Aldrich Pvt Ltd) in 3 different ratios, 100:0.5, 100:1, 100:1.5 (by volume) labelled as AgNP-0.5, AgNP-1 and AgNP-1.5, respectively. The resulting mixture was irradiated under direct sunlight; gradual colour changes were observed as a sign of nanosized silver particle formation. Every $30 \mathrm{~s}$, samples were subjected to kinetic-based UV studies for pinpointing the end time of nanoparticle formation and stability duration. The AgNPs were packed at the bottom by centrifuging for $15 \mathrm{~min}$ at $12000 \mathrm{rpm}$. The supernatant phase was filtered out, and the sedimented AgNPs were washed $3 \times$ with $10 \mathrm{ml}$ DD water.

\section{Coating on a glass slide using SILAR:}

The separated AgNPs were deposited as a thin films on a glass substrate using the SILAR technique. A deposition cycle of the $\mathrm{Ag}$ thin film consisted of 4 steps. First, the substrate was immersed in methanol dispersed Ag solution for $10 \mathrm{~s}$. Then the substrate was rinsed in methanol for $30 \mathrm{~s}$ to remove excess unabsorbed $\mathrm{Ag}$ ions. The deposition cycles were repeated 150 times. The primed thin films were characterized using diffraction analysis. Scanning electron microscopy (SEM) images were recorded using a microscope (Jeol JSM-6360) with $20 \mathrm{kV}$ voltage.

\section{Characterization of AgNPs:}

One millilitre of the nanoparticle mixture was periodically examined for completion of the bioreduction reaction of $\mathrm{Ag}+$ in aqueous solution, followed by addition of the methanol fraction of T. procumbens. Subsequent UV/Vis spectral scan read with the wavelengths of 250 to $600 \mathrm{~nm}$ in a spectrophotometer Beckman-Model No. DU-50, CA, USA, having a resolution of $1 \mathrm{~nm}$. UV/Vis spectra were saved at intervals of every $30 \mathrm{~s}$ up to $45 \mathrm{~min}$ and then once for every $30 \mathrm{~min}$. The absorbance peaks were analysed for the formation and degradation of NPs using the kinetic process.

The particle size of synthesized AgNPs was measured using the radiation scattering techniques (Malvern system). Dynamic light scattering (DLS) examines the size dispersal of particles (nanometer) in mixture with a scale ranging from submicron down to one. The measurement of particle size in Malvern system begins with the random movement of particles in a liquid due to the Brownian motion of particles in a sample using DLS and then interpreting a size using established concepts.

The dispersion stability of synthesized silver nanoparticle was measured by zeta potential. The Malvern zeta seizer 2000 used to calculate the Surface zeta potentials of ions. The Coulomb explosion engendered due to repelling of surface charged silver nanoparticle each other. It precludes the agglomerate tendency of the particle in solution. The surface potential and the dispersing liquid potential, which diverges according to the distance from the particle surface. The criteria of stability of NPs are measured when the values of zeta potential ranged from higher than $+30 \mathrm{mV}$ to lower than $-30 \mathrm{mV}$. Freeze dried AgNPs were dispersed in $50 \mathrm{ml}$ of DD water using $\mathrm{NaCl}$ as a suspending electrolyte solution $\left(2 \times 10^{-2} \mathrm{M}\right.$ $\mathrm{NaCl}$ ). The mixture was shaken for $30 \mathrm{~min}$, and the equilibrium $\mathrm{pH}$ was recorded.

Synthesized AgNPs-coated glass slide was subjected to $\mathrm{X}$-ray diffraction (XRD) pattern analysis to investigate both molecular, crystal structures, qualitative identification of metal ions and particle sizes. Unique diffraction pattern of synthesized AgNPs identified by paralleling the diffracted beams of the reference database in the joint committee on powder diffraction standards (JCPDS) library. Phase identification Match software (v3.6.2) was used to correlate the experimental pattern with the XRD reference database.

The AgNPs coated by SILAR method was used for SEM analysis. The thin AgNPs film images were developed in a Zeiss EVO-MA 10 SEM (Germany). The details of working condition (voltage, magnification) and size of the particle were included on the images itself. In addition, the edax potential of the AgNPs was measured and analysed.

The $200 \mathrm{kV}$, ultra-high resolution transmission electron microscope Jeol, model no. JEM 2100 HR with EELS used to record the morphology of the synthesized AgNPs. TEM module was prepared by dropping $5 \mu 1$ of the AgNPs solutions on carbon-coated copper grids and dried under the lamp.

The synthesized AgNPs were characterized using atomic force microscopy (AFM; NanoWizard II, JPK Instruments, Germany) for determining the size and 
morphology. The dispersed solution was prepared as a thin layer by dropping $100 \mu 1$ of the sample on a glass slide and allowed to dry for $5 \mathrm{~min}$. The AFM images were scanned with silicon cantilevers (CSC17, Mikro Masch, Estonia) in contact mode. The AFM images were processed using JPK data processing software (JPK Instruments, Germany).

Dynamic simulation studies and interaction studies were performed using the Biovia Discovery studio 2017 and Material studio 2017. Biovia Discovery Studio is a multi-dimensional CHARMm molecular mechanics simulation tool. The material studio is a complete modelling and simulation environment designed to allow predicting and understanding of the realistic model.

The compounds identified from the GC-MS studies were submitted to the Forcite molecular mechanic's tools. All the 2D molecules coordinates were converted to $3 \mathrm{D}$ atomistic document or a 3D Atomistic Trajectory document to investigate the potential energy surface, on which the atomic nuclei move. Their steps involved, running a Forcite calculation, which can be grouped as follows: once a 3D atomistic document has been defined, then the smart algorithm was selected with tolerance energy $0.001 \mathrm{kcal} / \mathrm{mol}$ and 500 iterations to measure geometric optimization. Consistentvalence force field (Cvff -version 2.4) assigned with an atom-based Van der Waals and electrostatic energy. Cubic spline truncation method with $12.5 \AA$ cutoff distance was fixed for energy calculation. The energy expression used in cvff is shown below Eqn.1, $\quad \mathrm{E}_{p o t}=\sum_{\mathrm{b}} \mathrm{D}_{\mathrm{b}}\left[1-\mathrm{e}^{-\alpha(\mathrm{b}-\mathrm{b}} 0^{2}\right]^{2}+\sum_{\theta} \mathrm{H}_{\theta}\left(\theta-\theta_{0}\right)^{2}+\sum_{\varphi} \mathrm{H}_{\varphi}(1-$ $\operatorname{SCOS}(\mathrm{n} \varphi)]+\sum_{x} \mathrm{H}_{x} \mathrm{X}^{2}+\sum_{\mathrm{b}} \sum_{\mathrm{b}^{\prime}} \mathrm{F}_{\mathrm{bb}}\left(\mathrm{b}-\mathrm{b}_{0}\right)\left(\mathrm{b}^{\prime}-\mathrm{b}_{0}^{\prime}\right)+\sum_{\theta} \sum_{\theta^{\prime}}(\theta-$ $\left.\theta_{0}\right)\left(\theta^{\prime}-\theta_{0}^{\prime}\right)+\sum_{b} \sum_{\theta} \mathrm{F}_{\mathrm{b} \theta}\left(\mathrm{b}-\mathrm{b}_{0}\right)\left(\theta-\theta_{0}\right)+\sum_{\varphi} \mathrm{F}_{\varphi \theta \theta^{\prime}} \cos \left(\theta-\theta_{0}\right)$ $\left(\theta^{\prime}-\theta_{0}^{\prime}\right)+\sum_{\mathrm{x}} \sum_{\mathrm{x}^{\prime}} \mathrm{F}_{\mathrm{xx}} \mathrm{xx}^{\prime}+\sum \varepsilon\left[\left(\mathrm{r}^{*} / \mathrm{r}\right)^{12}-2\left(\mathrm{r}^{*} / \mathrm{r}\right)^{6}\right]+\sum \mathrm{q}_{\mathrm{i}} \mathrm{q}_{\mathrm{j}} / \varepsilon \mathrm{r}_{\mathrm{ij}} .1$ to 4 terms referred to as the diagonal terms of the valence force field and epitomize the energy of deformation of bond lengths, bond angles, torsion angles, and outof-plane interactions, respectively. All the molecules include the same parameters measured silver, nitrate ion potential energies, and submitted for the orbital analysis using DFT.

The DFT protocol allowed calculating the energy or optimizing the geometry of the phytoconstituent of T. procumbens using the $\mathrm{DMol}^{3}$ calculation. The force field measure 3D atomic structures of each phytoconstituent were loaded into the Molecule Window of DS-2017. Further, the calculate energy protocol parameters were set to measure total energy,
HOMO energy, LUMO energy, dipole, atomic charges. The calculated molecular properties found in the Molecule tab of the Data Table View. If there are atomic properties such as fitted atomic charges, found in the Atom tab of the Data Table View. The isosurface of the electron density coloured by the electrostatic potential is added for up to the first 100 molecules. By default, the isovalue of the electron density is 0.03 , and the colouring scheme is spectrumRainbow1 with a range from -0.05 to 0.1 . The HOMO and LUMO can be visualized using scripts accessible from in the report. The positive phase of the molecular orbitals uses an isovalue of 0.01 and is coloured blue, while the negative phase uses an isovalue of -0.01 and is coloured red. Orbital nature of all the molecules, silver ion, and the nitrate ion, were analysed and discussed.

To identify the phytoconstituent, which is responsible for reducing $\mathrm{AgNO}_{3}$ to AGNPs, a realistic model was developed using the amorphous cell module in MS2017. The amorphous cell module constructs threedimensional periodic structures of molecular systems. The module physiques molecules in a cell by Monte Carlo fashion and minimizing close contacts between atoms, while confirming a realistic distribution of torsion angles for any given force field. All the 18 phytoconstituents and silver nitrate ions were packed into the cubic cell. The unit cell dimensions are determined by the mass density and the number of molecules and set automatically. The existing empty surface was filled with the water components in the mole ratio specified. The number of molecules is determined by the mass density, the volume and mass of the packing region, and set automatically.

After generating the amorphous cell, the location of the silver and nitrate ion with all the 18 phytoconstituent in an aqueous environment was analysed using the Adsorption Locator protocol. It finds the low energy adsorption orientation on both periodic and nonperiodic substrates of discrete systems. The adsorption locator module run with the 3 heating cycles, 100000 loading steps and optimize geometry. Stimulated annealing performed with adjusted Monte Carlo step size to measure overall system energies. The non-bonded interaction between the silver, nitrate ions were analysed.

Blends modules were used to measure the binding energies between the silver and nitrate ion with each phytoconstituent separately. The module calculates pair energy with the trajectories and atom-based nonbonded 
interaction energies were measured using $0.020 \mathrm{kcal} /$ mol energy bin width.

The functional group of capped phytoconstituent with AgNPs was identified by FTIR-potassium bromide $(\mathrm{KBr})$ pellet method in $(1: 100)$. IR spectrum was recorded on a Fourier transform infrared spectrometer Jasco FT/IR-6300 using transmittance mode operating at a resolution of $4 \mathrm{~cm}^{-1}$ (Jasco, Tokyo, Japan).

\section{Precipitation of silver by argentometric method:}

The separation of capped phytoconstituent from silver ion, complex formation principle was used. An equivalent amount of $\mathrm{KCN}$ was added to the dispersed AgNPs solution. The solution was allowed to settle down, and then a few drops of KCN were added slowly to the upper supernatant liquid to test the completeness of precipitation. The precipitate was separated by filtration. The remaining filtrate was subjected to highperformance thin layer chromatography (HPTLC) analysis.

HPTLC method was carried out using the mobile phase of benzene $(9 \mathrm{ml})$ :chloroform $(5 \mathrm{ml})$ :ethyl acetate $(8 \mathrm{ml})$ was selected based on a trial and error method. Ten microliters each with band length of $5 \mathrm{~mm}$ separated filtrate from synthesized AgNPs solution (after precipitation) was applied on TLC aluminium sheets silica gel 60 F 254 (Merck) in Camag HPTLC system, which was programmed through winCATS software. The chromatograms were developed in Camag twin trough chamber saturated with mobile phase solvent for $20 \mathrm{~min}$ up to the distance of $80 \mathrm{~mm}$. The bands scanned at both 254 and $366 \mathrm{~nm}$ by deuterium and tungsten lamp with slit dimension of the $6.0 \times 0.45$ macro. The data was integrated through the winCATS Planar Chromatography Manager.

\section{RESULTS AND DISCUSSION}

The GC-MS retention times and interpretation of mass spectral analysis of the methanol fraction of aqueous extract of $T$. procumbens revealed the presence of 18 compounds. Table 1 showed the details of the compounds identified. The structural characterization indicated that the GC-MS found 10 flavonoids, 6 alkaloids, a hydroxycinnamate, carotenoids and a benzoic acid derivative (Table 1).

When the methanol fraction was mixed in different ratios with $\mathrm{AgNO}_{3}$ and irradiated in sunlight, within $1 \mathrm{~min}$, the colour of the solution began to change from colourless to dark violet, showing the nucleation of AgNPs. Among the 3 mixtures, the AgNP-0.5 and AgNP-1 formed stable particles without micelle formation. It is an ultrafast method, which as reported by other researchers worked with different plant systems ${ }^{[18]}$.

$\mathrm{UV} / \mathrm{Vis}$ spectroscopy is the most convenient methods for characterizing the optical response of AgNPs and sensitive to the materialization of colloidal metal NPs, due to their strong surface plasmon resonance (SPR). The results of the colour and absorption intensity revealed that the colloidal samples AgNP-1 were

TABLE 1: GC-MS ANALYSES OF METHANOL FRACTION OF AQUEOUS EXTRACT OF T. PROCUMBENS LEAVES

\begin{tabular}{lcccc}
\hline RT $(\mathrm{min})$ & Compound & Compound nature & Molecular formula & Molecular weight (g/mol) \\
\hline 9.133 & Sparteine & Alkaloid & $\mathrm{C}_{15} \mathrm{H}_{26} \mathrm{~N}_{2}$ & 234.387 \\
11.072 & Lupanine & Alkaloid & $\mathrm{C}_{15} \mathrm{H}_{24} \mathrm{~N}_{2} \mathrm{O}$ & 248.37 \\
14.153 & Caffeic acid & Hydroxycinnamates & $\mathrm{C}_{9} \mathrm{H}_{8} \mathrm{O}_{4}$ & 180.159 \\
14.367 & Vanilic acid & Benzoic acid & $\mathrm{C}_{8} \mathrm{H}_{8} \mathrm{O}_{4}$ & 168.148 \\
16.83 & Naringenin & Flavonoid & $\mathrm{C}_{15} \mathrm{H}_{12} \mathrm{O}_{5}$ & 272.256 \\
17.1 & Biochanin & Flavonoid & $\mathrm{C}_{16} \mathrm{H}_{12} \mathrm{O}_{5}$ & 284.267 \\
19.177 & Epicatechin & Flavonoid & $\mathrm{C}_{15} \mathrm{H}_{14} \mathrm{O}_{6}$ & 290.271 \\
21.408 & Quercetin & Flavonoid & $\mathrm{C}_{15} \mathrm{H}_{10} \mathrm{O}_{7}$ & 302.238 \\
22.852 & (-)-Epicatechin-3-gallate & Flavonoid & $\mathrm{C}_{22} \mathrm{H}_{18} \mathrm{O}_{10}$ & 442.376 \\
23.535 & $(-)$-Epigallocatechin-3-gallate & Flavonoid & $\mathrm{C}_{22} \mathrm{H}_{18} \mathrm{O}_{11}$ & 458.375 \\
24.792 & Lutein & Carotenoids & $\mathrm{C}_{40} \mathrm{H}_{56} \mathrm{O}_{2}$ & 568.886 \\
25.797 & Baicalein & Flavonoid & $\mathrm{C}_{15} \mathrm{H}_{10} \mathrm{O}_{5}$ & 270.24 \\
26.642 & Nobiletin & Flavonoid & $\mathrm{C}_{21} \mathrm{H}_{22} \mathrm{O}_{8}$ & 402.399 \\
26.85 & Baicalin & Flavonoid & $\mathrm{C}_{21} \mathrm{H}_{18} \mathrm{O}_{11}$ & 446.364 \\
27.487 & Silymarin & Flavonoid & $\mathrm{C}_{25} \mathrm{H}_{22} \mathrm{O}_{10}$ & 482.441 \\
29.34 & Emetine & Alkaloid & $\mathrm{C}_{29} \mathrm{H}_{40} \mathrm{~N}_{2} \mathrm{O}_{4}$ & 480.649 \\
30.683 & Thalicarpin & Alkaloid & $\mathrm{C}_{41} \mathrm{H}_{48} \mathrm{~N}_{2} \mathrm{O}_{8}$ & 696.841 \\
32.558 & Paclitaxel & Alkaloid & $\mathrm{C}_{47} \mathrm{H}_{51} \mathrm{NO}_{14}$ & 853.918 \\
\hline
\end{tabular}


stable up to $48 \mathrm{~h}$ and the absorbance was at $412 \mathrm{~nm}$, while the addition of $1 \mathrm{ml}$ of the methanol fraction into $100 \mathrm{ml}$ of silver nitrate solution. Such observation gave preliminary indication regarding the shape and size of AgNPs. Addition of $1.5 \mathrm{ml}$ extract showed the shifts towards higher wavelength $582.15 \mathrm{~nm}$ and unstable of AgNPs.

A kinetic study of the reduction process shows (figs. 1a, b) that the synthesis of AgNPs started within $30 \mathrm{~s}$ of addition and reached a plateau after $30 \mathrm{~min}$. In the initial $30 \mathrm{~s}$, there is gradual rise in absorbance followed by a slow increase in the absorbance values, which finally become linear after $90 \mathrm{~min}$ for thus suggesting very fast reaction kinetics of SNs synthesis from AgNP-1 compared to other mixtures.

The blanks did not show any colour change even after two days. It signified that sunlight plays an important role in the biosynthesis of $\mathrm{AgNPs}^{[19]}$. Sunlight enriched the energy of $\mathrm{Ag}+$ ion to the sharing of electrons in the process ${ }^{[20]}$. A plot is drawn between the percent absorbance vs. time $t$ as a pseudo-first order rate constant (kobs), the same is fitted in to the Eqn., $A t=A_{\mathrm{f}}+\left(A o-A_{\mathrm{f}}\right) \exp (-k \mathrm{obs} t)$, where $A o$ and $A_{\mathrm{f}}$ are the initial and final absorbance" AgNPs, respectively ${ }^{[21,22]}$.

The intensity distribution and polydispersity index (PDI) of primary result from DLS showed the distribution width of AgNPs formed using methanol fraction of $T$. procumbens. Fig. 1c exposed that the size and the associated relative percentage of particles distribution in AgNPs-1 by the intensity of light scatter. The $Z$ average of the 1-min sample of 10:1 ml ratio mixture found to be $63.30 \mathrm{~d}$. nm with $\mathrm{s}$. dev of 12.55. PDI of 0.041 clearly describes the sample less polydispersed. The fit of the geometrically spaced actual data points by the cumulates (fig. 1c) analysis from which the $\mathrm{z}$-average diameter and PDI verified the good quality data, and there is no presence of large or sediment particles. The excess addition of methanol fraction $(10: 1.5 \mathrm{ml})$ showed the greater particle size and very polydispersity of AgNPs.

To further gain insights into the stability of the engineered $\mathrm{AgNPs}$, zeta potential was measured ${ }^{[23,24]}$. Herein, the $\zeta$ values wide-ranging from 15.5, 38.5 and $68.3 \mathrm{Mv}$ for the AgNPs-0.5, AgNPs-1 and AgNPs-1.5, respectively. It suggested that the surface of the NPs is positively charged and dispersed in the neutral medium. The negative value of electrophoretic mobility confirms the repulsion forces. It quantified by the small frequency shift of the light. An articulate laser source (laser) scattered by the charged particle that is moving in a peripheral electric field between the particles proved that NPs are very stable electrophoretic mobility distribution. Fig. 1d showed the average electrophoretic mobility and zeta potential values of the synthesized AgNPs.

After 125 cycles in SILAR, the good-quality AgNPs film was deposited and quantified by the XRD pattern. The grey colored, thin films well-adhered to the glass (silicon) substrate. Fig. 1e showed the diffractograms of the AgNPs-1 film with glass substrates and the Ag particle is amorphous and fine grains in nature. The Ag (111) substrate are strongly surfaced along with a hexagonal crystal structure of the nanoparticle. The crystal data entry $96-210-5382$ pattern ${ }^{[25]}$ was matched well with experimental value with the figure-of-merit value of 0.8369 . Fig. 1e shown four distinct (111), (200), (220) and (311) reflections at 38.12, 44.38, 64.45 and 77.41 indicated the metallic silver. The hexagonal assembly of AgNPs conformed by vociferous and stout diffraction peak cantered at 38.12, which referenced to the (111) reflection and close fit. The $69.25 \mathrm{~nm}$ crystallite size of the AgNPs assessed by the DebyeScherrer formula, which was greater than the mean size of the TEM analysis.

From the integrated results of SEM, the sample, which consisted of $1 \mathrm{ml}$ of the methanol fraction in silver nitrate solution, consisted of $63.35 \mathrm{~nm}$ sized particles (fig. 2a). Also, the agglomerated NPs formed by the mixture containing $1.5 \mathrm{ml}$ of extract. EDX analysis was employed, it was perceived that $60 \%$ weight of the product is Ag nanofilm and $40 \%$ silicon ion existed (substrate). The help of TEM clarified the outline shape and dimension of the particles (fig. 2b). Carbon-coated copper grid with dried AgNPs solution was evaluated using TEM images. The micrograph of TEM result proposed the sizes of the synthesized AgNPs were around $74 \mathrm{~nm}$. The dimension analysis labelled the synthesized AgNPs was hexagonal. Non-contact mode of AFM surface analysis reports shown in figs. 2c-f. The $1 \times 1 \mu \mathrm{m}$ area of surface analysed and the images revealed that the topography of the particle is properly systematic construction for the (fig. 2e) sample-3 showed aggregated particle.

Molecular modelling is a well-adapted technique to investigate the organization of the molecular level interaction studies ${ }^{[26-28]}$. Here a new correlated approach was devoted to pointing out the phytoconstituent of the methanol fraction of $T$. procumbens, which is 

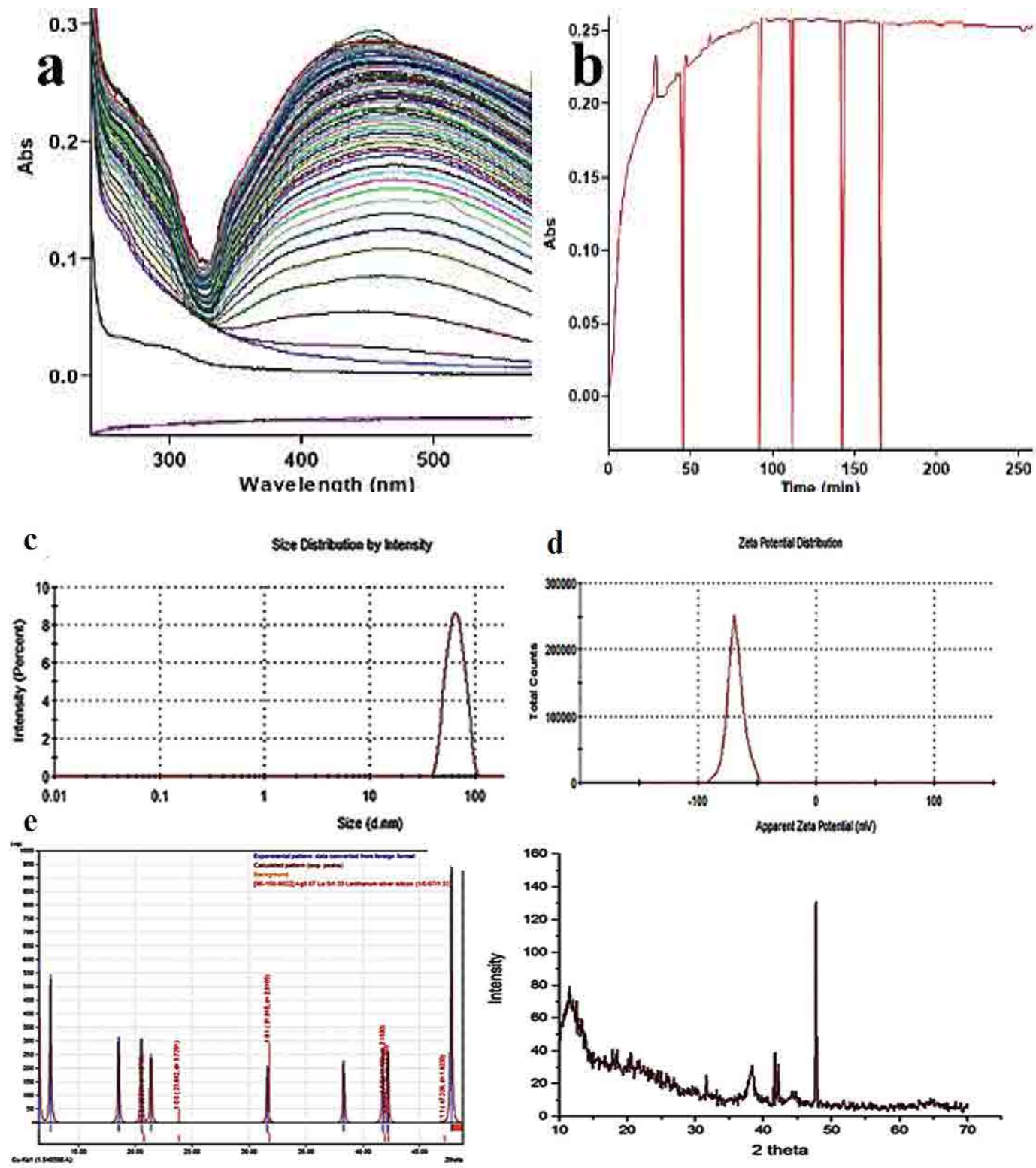

Fig. 1: UV spectrum (a, b), DLS report, zeta analyzer report sheet and XRD-pattern of sample AgNP-1 (a) 2D spectrum, (b) kinetic analysis graph, (c) DLS report, (d) zeta analyzer report, (e) XRD-pattern

responsible for shaping and stabilizing the AgNPs. Phytoconstituent were identified using the interaction, energy, binding energy, HOMO, LUMO orbital and $\mathrm{Dmol}^{3}$ calculation. Modelling protocols begin with potential energy calculation (forcefield) to molecular dynamics experiments executed on immeasurable solids with periodic boundary conditions.

The classical force field-based simulation was performed on both molecular and periodic systems ${ }^{[29,30]}$. The protocol run resulted in the energy calculated and minimized 18 molecules for future studies (Table 2). The initial potential energy brought to the local minima of $-27.59 \mathrm{kcal} / \mathrm{mol}$ and the $\mathrm{VdW}$; electrostatic values range from -5.03944 to 7.8936 and -39.65714 to $5.71059 \mathrm{kcal} / \mathrm{mol}$, respectively. All the molecules were minimized using the 1000 steps of conjugated gradient followed by 1000 steps of steepest gradient method. Forcite geometric optimization level for all the phytoconstituents energy and convergence, revealed that all molecules energy brought to the local minima to make molecules stable in the complex system $^{[31]}$.

DFT protocol used to study the chemical reactivity pattern between the phytoconstituent and silver nitrate ions. The tabled reactivity descriptors were added in the properties table of each phytoconstituent and analyzed (Table 3). The density functional quantum mechanics method in $\mathrm{DMol}^{3}$ calculate the energy of the molecules. The total energy of each system calculated using optimized variations ${ }^{[32]} E_{\mathrm{t}}$ respect to variations 


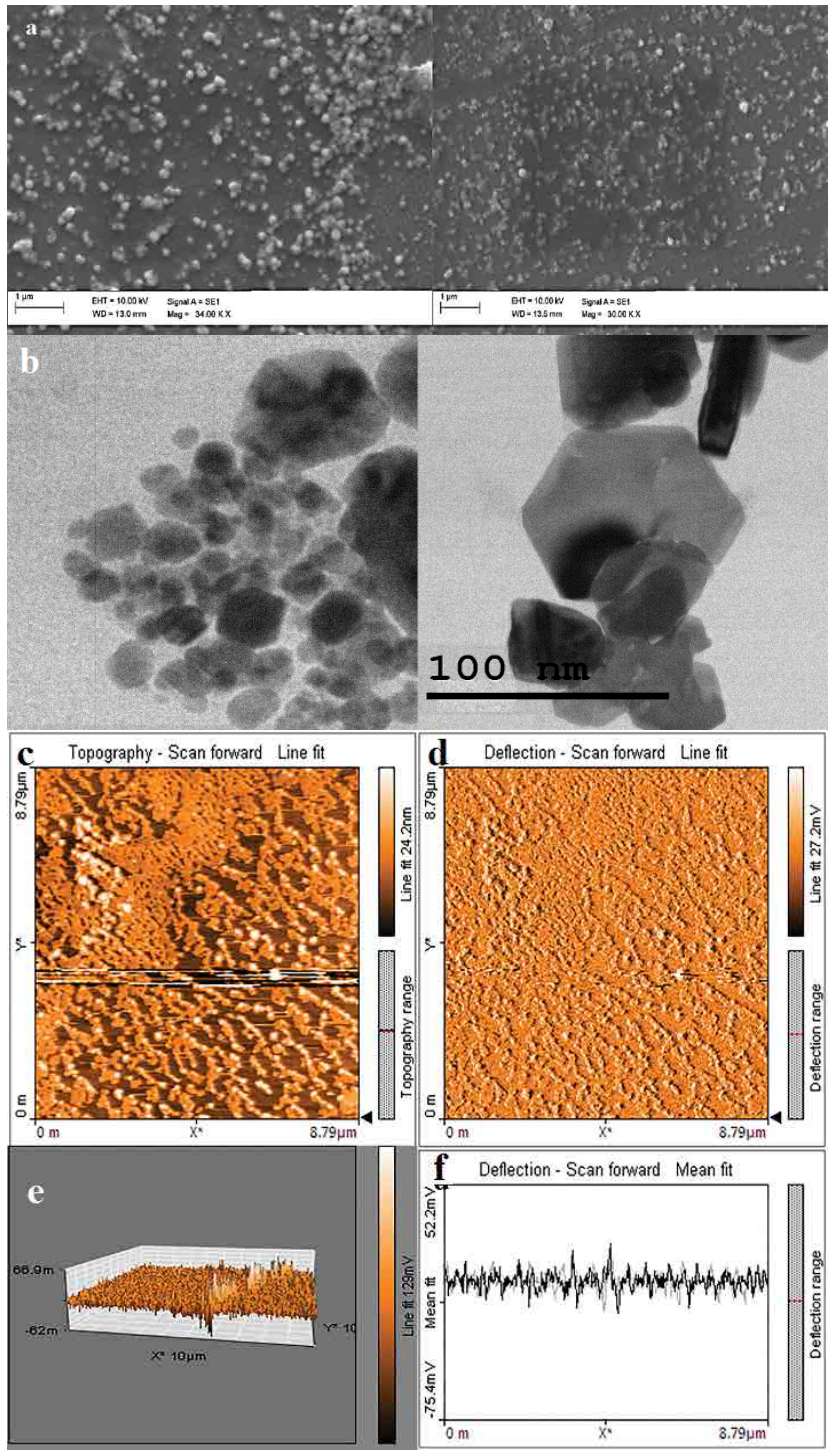

Fig. 2: SEM, TEM and AFM analysis of synthesized AgNPs-1 (a) SEM (magnification 34.00, $30.00 \mathrm{kx}$ ), (b) TEM, (c-f) AFM: c. topography, d. deflection, e. 3D image of deposited AgNP-1, f. deflection mean fit in $\rho$, subject to orthonormality constraints by using Eqn. $2, \mathrm{E}_{\mathrm{t}}[\rho]=\sum_{\mathrm{i}}\left\langle\varphi_{\mathrm{i}}+\left|-\nabla^{2} / 2\right| \varphi_{\mathrm{i}}\right\rangle+\left\langle\rho\left(\mathrm{r}_{1}\right)\left[\varepsilon_{\mathrm{xc}}\left[\rho\left(\mathrm{r}_{1}\right)+\left(\mathrm{V}_{\mathrm{e}}\left(\mathrm{r}_{1}\right) / 2-\right.\right.\right.\right.$ $\left.\left.\mathrm{V}_{\mathrm{N}}\right]\right\rangle+\mathrm{V}_{\mathrm{NN}}$. The higher total energy of the Ag ion found to be $-5194.8482 \mathrm{kcal} / \mathrm{mol}$ making the ability to form non-bonded interaction with the phytoconstituent.

The cubic amorphous cell boundary composed of the 18 phytoconstituents and $\mathrm{n}$ number of silver, nitrate ion and a varying number of water molecules in the isosurface of the periodic boundary. In order to explicate the interaction mechanism between the phytoconstituent, silver, nitrate ions and a water molecule in the amorphous regions, adsorption location and binding energies were analysed between the formed complexes ${ }^{[33]}$ (fig. 3). All interaction pattern was analysed to point out the phytoconstituent, which is answerable for the reduction and stabilization of silver ion.

Adsorption locator investigates that low energy adsorption sites and preferential adsorption site of the individual complex (phytoconstituent-Ag) in both periodic and nonperiodic substrates ${ }^{[34,35]}$. This module generated 10 different coordinated atoms in each phytoconstituent, which would form the stable interaction between Ag or nitrate ion. Based on the result of adsorption energy (Table 4) between ions and phytoconstituent showed baicalin and $\mathrm{Ag}$ ion $(-39.58290904 \mathrm{kcal} / \mathrm{mol})$ for the most favorable and stable conformation in the orientation space. Fig. $2 b$ explained the energy distribution and total energy of the Ag and baicalin complex.

The compatibility of $\mathrm{Ag}$ ion and phytoconstituent mixtures was perceived by using modified Flory-
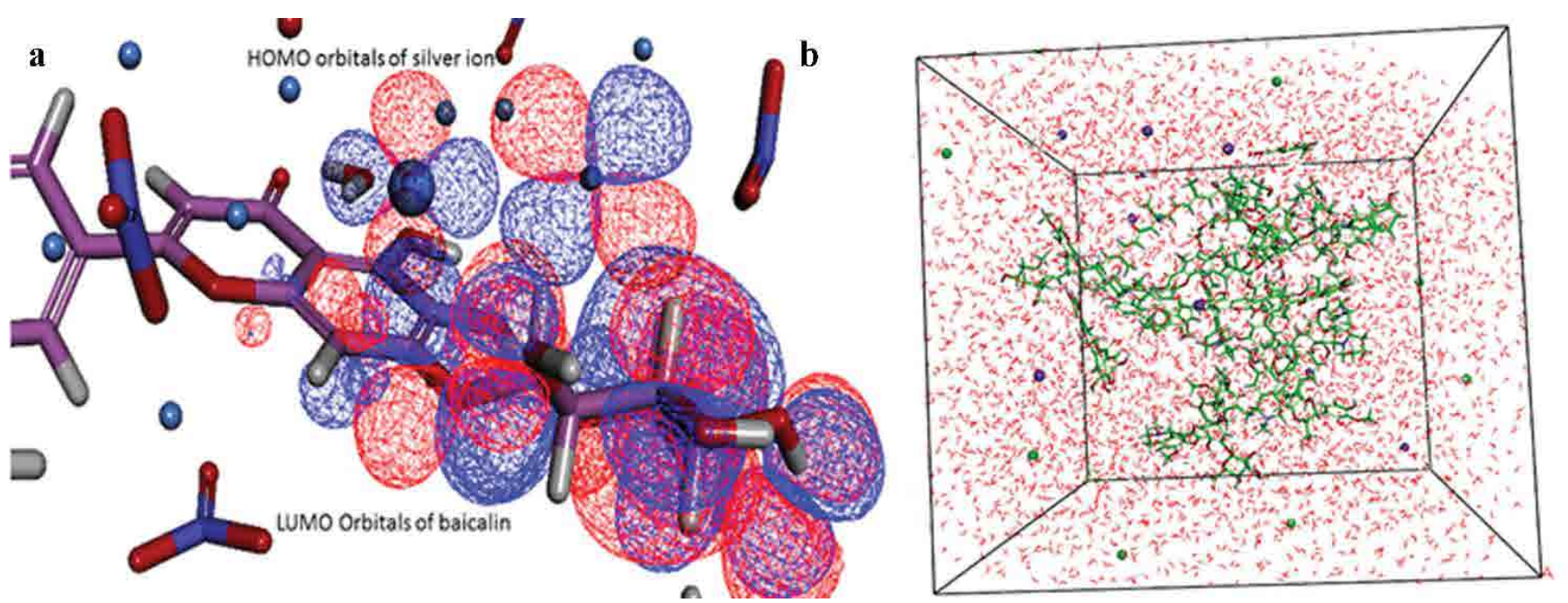

Fig. 3: Special arrangement of HOMO and LUMO orbitals and cubic amorphous cell

a. special arrangement of HOMO and LUMO orbitals of Ag-baicalin phytoconstituents, b. cubic amorphous cell for the mixture of silver nitrate and methanol fraction of T. Procumbens extract 
TABLE 2: FORCITE ENERGY ANALYSIS FOR PHYTOCONSTITUENT FROM METHANOL FRACTION OF T. PROCUMBENS

\begin{tabular}{|c|c|c|c|c|c|}
\hline Name & $\begin{array}{l}\text { Potential energy } \\
(\mathrm{kcal} / \mathrm{mol})\end{array}$ & $\begin{array}{c}\text { Van der Waals } \\
\text { energy }(\mathrm{kcal} / \mathrm{mol})\end{array}$ & $\begin{array}{l}\text { Electrostatic energy } \\
(\mathrm{kccl} / \mathrm{mol})\end{array}$ & $\begin{array}{l}\text { Initial RMS gradient } \\
(\mathrm{kcal} /(\mathrm{mol} \times \mathrm{A})\end{array}$ & $\begin{array}{l}\text { Final RMS gradient } \\
(\mathrm{kcal} /(\mathrm{mol} \times \mathrm{A})\end{array}$ \\
\hline Epicatechin & -1.02019 & 1.89605 & -11.92568 & 36.07911 & 0.09069 \\
\hline Quercetin & 11.77143 & 6.19033 & -2.36605 & 39.32582 & 0.06521 \\
\hline Biochanin & 22.09045 & 7.89363 & 5.71059 & 41.42223 & 0.09012 \\
\hline Naringenine & -0.40675 & 1.81625 & -8.37517 & 38.76939 & 0.09175 \\
\hline $\begin{array}{l}\text { Epicatechin } \\
\text { gallate }\end{array}$ & -21.28973 & 1.90259 & -37.17458 & 37.57906 & 0.09857 \\
\hline Lupanine & 30.3964 & -5.03944 & -0.42644 & 18.88655 & 0.0955 \\
\hline Lutein & 103.7392 & 0.92074 & -3.51414 & 36.31153 & 0.09764 \\
\hline Caffic acid & 0.6652 & 2.43084 & -4.23359 & 34.67402 & 0.08092 \\
\hline Vanillic acid & 2.70859 & 2.41144 & -2.73987 & 35.85937 & 0.08886 \\
\hline Sparteine & 64.67449 & -2.82591 & 2.02034 & 22.02862 & 0.09287 \\
\hline $\begin{array}{l}\text { Epigallocatechin } \\
\text { gallate }\end{array}$ & -27.59193 & -0.63506 & -39.65714 & 36.58475 & 0.09207 \\
\hline Baicalein & -2.26993 & 2.93316 & -10.96664 & 39.47943 & 0.09763 \\
\hline Nobiletin & 37.82017 & 2.14316 & 4.15778 & 66.47018 & 0.09872 \\
\hline Paclitaxel & 168.77535 & 5.03954 & -24.37265 & 40.88184 & 0.08987 \\
\hline Thalicarpin & 58.55352 & 3.57435 & 3.34356 & 20927.715 & 0.08955 \\
\hline Emetine & 39.2623 & -0.06015 & 0.28047 & 26.13741 & 0.08559 \\
\hline Silymarin & 11.63992 & 4.43593 & -8.17302 & 263.74652 & 0.09577 \\
\hline Baicalin & 24.65688 & 0.46483 & -12.50534 & 39.08043 & 0.07934 \\
\hline
\end{tabular}

TABLE 3: DENSITY FUNCTIONAL THEORY (DFT) ANALYSIS OF MOLECULES

\begin{tabular}{lcccccc}
\hline Name & $\begin{array}{c}\text { Total energy } \\
\mathbf{( H a )}\end{array}$ & $\begin{array}{c}\text { Binding } \\
\text { energy }(\mathrm{Ha})\end{array}$ & $\begin{array}{c}\text { HOMO energy } \\
(\mathbf{H a})\end{array}$ & $\begin{array}{c}\text { LUMO energy } \\
(\mathrm{Ha})\end{array}$ & $\begin{array}{c}\text { Band Gap } \\
\text { energy }(\mathrm{Ha})\end{array}$ & $\begin{array}{c}\text { Dielectric } \\
\text { energy }(\mathrm{Ha})\end{array}$ \\
\hline Biochanin & -985.185 & -7.34694 & -0.200952 & -0.095487 & 0.1054644 & -0.039054 \\
Naringenine & -947.451 & -7.07992 & -0.214557 & -0.101526 & 0.1130308 & -0.030549 \\
Emetine & -1526.69 & -14.6419 & -0.178771 & -0.049439 & 0.1293327 & -0.029980 \\
Epicatechin gallate & -1589.15 & -11.0219 & -0.205938 & -0.093893 & 0.112045 & -0.055035 \\
Lupanine & -764.268 & -7.98646 & -0.176292 & -0.026618 & 0.149674 & -0.019002 \\
Lutein & -1693.38 & -18.8599 & -0.175063 & -0.113427 & 0.0616356 & -0.037869 \\
Caffic acid & -643.667 & -4.53737 & -0.206108 & -0.108234 & 0.0978738 & -0.033820 \\
Vanillic acid & -605.894 & -4.23175 & -0.213927 & -0.090114 & 0.1238139 & -0.026485 \\
Sparteine & -690.634 & -7.92072 & -0.154594 & 0.0216662 & 0.1762609 & -0.007533 \\
Epigallocatechin gallate & -1663.90 & -11.2443 & -0.205304 & -0.092610 & 0.1126942 & -0.056185 \\
Baicalein & -946.254 & -6.83993 & -0.209582 & -0.106193 & 0.1033883 & -0.036988 \\
Nobiletin & -1404.06 & -10.5337 & -0.202596 & -0.105755 & 0.0968406 & -0.031570 \\
Paclitaxel & -2906.15 & -23.3200 & -0.197122 & -0.115216 & 0.0819062 & -0.077398 \\
Thalicarpin & -2283.64 & -20.0633 & -0.16866 & -0.086082 & 0.0825778 & -0.044892 \\
Silymarin & -1704.79 & -12.3429 & -0.206257 & -0.110410 & 0.095847 & -0.047353 \\
Baicalin & -1626.07 & -10.8787 & -0.213904 & -0.106295 & 0.1076088 & -0.060505 \\
Ag & -5194.84 & 0.16804 & -0.368020 & -0.177656 & 0.1903637 & -0.113209 \\
Nitrate ion & -278.574 & -0.86864 & -0.199034 & -0.094425 & 0.104609 & -0.104765 \\
Epicatechin & -1023.36 & -7.50974 & -0.202757 & -0.050566 & 0.1521904 & -0.056185 \\
Quercetin & -1095.75 & -7.28783 & -0.197711 & -0.103255 & 0.0944563 & -0.036988 \\
\hline
\end{tabular}

Huggins blend model and role property ${ }^{[36]}$. Blends distinguish the components by using the role property. In each mixture considered by Blends, one component has a base role, and the other has a screen role ${ }^{[37]}$. These abbreviated as $b$ and $s$, respectively. Table 5 describes the binding energy (energy of interaction) of each phytoconstituent (base) with individual screen atoms such as Ag, nitrate, and water. Blends generated a large number of molecular orientations and calculated the pair interaction energies of each configuration. Excluded-volume constraints also included making van der Waals surfaces are in contact.

Interactions between the $\mathrm{Ag}$ ion and phytoconstituent described by the non-bond interactions such as 


\begin{tabular}{lccccc}
\hline Structures & Total energy & $\begin{array}{c}\text { Adsorption } \\
\text { energy }\end{array}$ & $\begin{array}{c}\text { Rigid } \\
\text { adsorption energy }\end{array}$ & $\begin{array}{c}\text { Deformation } \\
\text { energy }\end{array}$ & Ag:dEad/dNi \\
\hline Epicatechin & -506.598483 & -1141.10 & -511.049834 & -630.046869 & -52.2186893 \\
Quercetin & -484.393927 & -1118.89 & -489.270790 & -629.621357 & -32.1446736 \\
Biochanin & -477.927904 & -1112.43 & -483.472317 & -628.953807 & -32.1016302 \\
Naringenin & -481.513854 & -1116.01 & -485.829962 & -630.182111 & -39.2038244 \\
Caffeic acid & -473.452132 & -1107.95 & -477.782773 & -630.167579 & -38.4250120 \\
Vanillic acid & -466.610885 & -1101.11 & -469.721989 & -631.387115 & -44.4942142 \\
Nobiletin & -486.565695 & -1121.06 & -491.467615 & -629.596300 & -38.6512777 \\
Baicalein & -504.704943 & -1139.20 & -512.462847 & -626.740317 & -39.0882815 \\
Epigallocatechin gallate & -466.318561 & -1100.82 & -470.110932 & -630.705849 & -36.8157679 \\
lutein & -432.391636 & -1066.89 & -435.411728 & -631.478127 & -39.2602435 \\
Epicatechin gallate & -486.891175 & -1121.39 & -491.907245 & -629.482149 & -42.5170538 \\
Sparteine & -469.637443 & -1104.14 & -473.415804 & -630.719859 & -48.8462603 \\
Lupanine & -471.858585 & -1106.36 & -477.761440 & -628.595365 & -37.3887560 \\
Pacilitaxal & -435.910963 & -1070.41 & -442.585667 & -627.823515 & -30.4024871 \\
Thalicarpin & -449.959272 & -1084.46 & -453.259172 & -631.198320 & -20.9771313 \\
Emetine & -486.532052 & -1121.03 & -490.406864 & -630.623407 & -43.1414749 \\
Silymarin & -453.281996 & -1087.78 & -457.578645 & -630.201571 & -36.3289917 \\
Baicalin & -464.992016 & -1099.49 & -468.634172 & -630.856063 & -39.5829090 \\
\hline
\end{tabular}

TABLE 5: BINDING ENERGIES OF INDIVIDUAL BASE AND SCREEN COMPLEX USING BLENDS

\begin{tabular}{|c|c|c|c|c|c|}
\hline Complex & Ebs $\min$ & Ebs avg & Complex & Ebs min & Ebs avg \\
\hline Epicatechin-Ag & -10.285 & -4.8086 & Pacilitaxal-nitrate & -5.5812 & -1.96837 \\
\hline Epicatechin-nitrate & -6.2535 & -1.8814 & Pacilitaxal-water & -6.9284 & -0.65606 \\
\hline Epicatechin-water & -4.9560 & -0.6402 & Silymarin-Ag & -0.6515 & -0.31769 \\
\hline Baicalin-Ag & -0.6307 & -0.3060 & Silymarin-nitrate & -3.6213 & -0.94509 \\
\hline Baicalin-nitrate & -2.8821 & -0.9146 & Silymarin-water & -4.7373 & -0.67226 \\
\hline Baicalin-water & -4.6431 & -0.6637 & Thalicarpin-Ag & -0.9130 & -0.32885 \\
\hline Quercetin-Ag & -10.711 & -5.3337 & Thalicarpin-nitrate & -3.9535 & -0.95961 \\
\hline Quercetin-nitrate & -5.7018 & -2.0628 & Thalicarpin-water & -5.4331 & -0.71269 \\
\hline Quercetin-water & -5.1657 & -0.7081 & Biochanin-Ag & -0.6011 & -0.28504 \\
\hline Naringenin-Ag & -9.3815 & -4.9592 & Biochanin-nitrate & -3.0161 & -0.84971 \\
\hline Naringenin-nitrate & -5.5777 & -1.9487 & Biochanin-water & -5.0258 & -0.6027 \\
\hline Caffeic acid-water & -4.8413 & -0.5998 & Pacilitaxal-Ag & -10.904 & -4.80002 \\
\hline vanillic acid-Ag & -8.5936 & -4.3021 & Epicatechin gallate-Ag & -13.831 & -5.22715 \\
\hline vanillic acid-nitrate & -4.5721 & -1.6409 & Epicatechin gallate-nitrate & -6.2076 & -2.05872 \\
\hline vanillic acid-water & -4.5000 & -0.5544 & Epicatechin gallate-water & -5.3469 & -0.68871 \\
\hline Nobiletin-Ag & -11.682 & -5.1217 & Sparteine-Ag & -7.7436 & -3.54125 \\
\hline Nobiletin-nitrate & -6.5659 & -1.9378 & Sparteine-nitrate & -4.2663 & -1.39444 \\
\hline Nobiletin-water & -4.8934 & -0.6657 & Sparteine-water & -4.2088 & -0.47395 \\
\hline Baicalein-Ag & -10.417 & -5.2550 & Lupanine-Ag & -9.5985 & -3.6466 \\
\hline Baicalein-nitrate & -5.5465 & -2.0331 & Lupanine-nitrate & -4.9641 & -1.43337 \\
\hline Baicalein-water & -5.3713 & -0.6926 & Lupanine-water & -5.4754 & -0.49445 \\
\hline Lutein-Ag & -8.3436 & -4.7714 & Lutein-water & -4.1956 & -0.64583 \\
\hline Lutein-nitrate & -4.8538 & -1.9336 & & & \\
\hline
\end{tabular}

attractive interactions, traditional interactions (classic hydrogen bonds) as well as weak hydrogen bonds with carbon donors. In addition to attractive forces, and repulsive interactions also monitored. Figs. $2 \mathrm{c}-\mathrm{f}$ and 4 explained the bond formed between the more stable complex of baicalin and Ag ion. IR spectrum of the synthesized silver nanoparticle conformed the capping compound contains the broad and less transmittance peak at $3391.21 \mathrm{~cm}^{-1}$ also $\mathrm{sp}^{3} \mathrm{C}-\mathrm{H}$ stretch at 2920.66 and $2851.24 \mathrm{~cm}^{-1}$. The peak at $\mathrm{C}=\mathrm{O} 1735.62 \mathrm{~cm}^{-1}$, aromatic $\mathrm{C}=\mathrm{C}$ stretch at $1626.66 \mathrm{~cm}^{-1}$ and the $\mathrm{Sp}^{3}$ bending peaks at $933.37 \mathrm{~cm}^{-1}$ confirmed that baicalin is capping the surface of the synthesized AgNPs. HPTLC analysis of the dispersed 


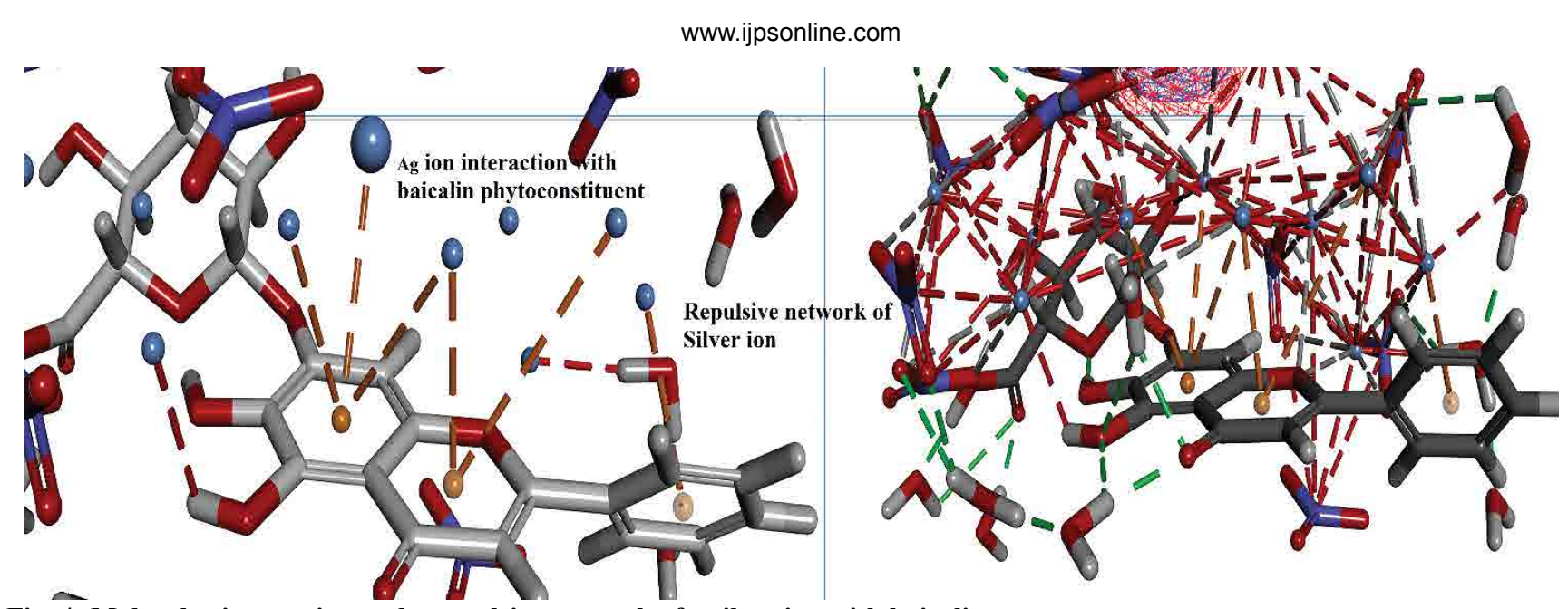

Fig. 4: Molecular interaction and a repulsive network of a silver ion with baicalin

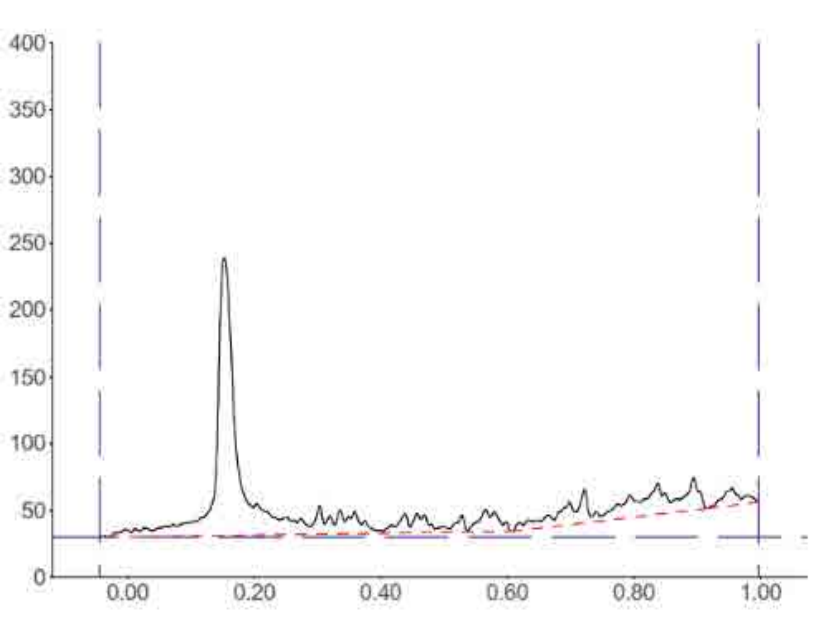

Fig. 5: HPTLC peak of silver reducing phytoconstituent

AgNPs solution after precipitation of $\mathrm{Ag}$ using the Mohr's method. The result of HPTLC analysis confirmed the major peak at $70.65 \%$ of the area with $\mathrm{Rf}$ start from 0.11 and end at 0.20 (fig. 5). This result confirmed the solution contained a single compound, which is responsible for the formation and shaping of the AgNPs.

Bioengineering of AgNPs using plant extracts is an ultra-fast growing field due to its eco-friendly nature. In this pathbreaking approach shown here, the new way to study molecular level insight of AgNPs formation by plant phytoconstituents. The colour change of the NPs mixture was due to surface plasmon vibrations, nature of phytoconstituent, size and shape of the metal particles ${ }^{[38]}$. In addition, these colour changes instigated by the interface of the electric field of light with confined surface electron charges within the particles, which triggered communal oscillation of the conduction electrons concerning the nucleus ${ }^{[39,40]}$. Typically, the materialization of nano-sized silver particles was inveterate by the appearance of shrill
SPRs in the range of $350-600 \mathrm{~nm}^{[41,42]}$. Based on the mixture ratio, absorption spectra (fig. 1) of AgNPs displayed bands, which differ in their $\lambda$ max and SP band intensities.

The DFT analysis, clearly explained the aptness of a complex between phytoconstituent and $\mathrm{Ag}$ ion. Measurement and analysis of HOMO and LUMO orbitals, as well as energy, showed that the silver ion (HOMO -0.3680202, LUMO-0.1776565) possessed higher HOMO orbitals to gain the electron. Examining the orbitals and energy of each phytoconstituent resulted in baicalin atoms found that (LUMO0.1062958 ) ability to donate the electrons and forming a complex with the Ag ions ${ }^{[43,31]}$. Indeed, this confirmed the superior stability of complexes associated with other phytoconstituent (fig. 3). Lower values of HOMO $-0.2139046 \mathrm{kcal} / \mathrm{mol}$, directed that baicalin receive electrons and less affinity as an electrophobic center. The HOMO energies of other phytoconstituent were higher than baicalin, which deteriorated from the electron donation with the $\mathrm{Ag}$ ion.

The negative values of binding energy and orbital potentials signifies more stability of complex systems. HOMO and LUMO gap also assess the stability of Ag complex with shifting conformation and orientation conferring to the principle ${ }^{[4]}$. As marked from Table 3, the band gap energy values increased on Ag-baicalin complex. Likewise, the code of minimum electrophilicity principle consigned that chemical stability of Ag ion complex inversely correlated to electrophilicity of each atom. Binding of phytoconstituent with silver cation produces a more significant value of energy -0.0605054 and lower energy of baicalin (-0.1132099) that specify it is easy for relocating charge. 
Moreover, higher negative values of baicalin indicate substantial global stability than other phytoconstituents. Subsequently, the Ag ion complex formation with other phytoconstituent was not auspicious on solvation. Overall, $\mathrm{Ag}^{+}$cation binding with baicalin was favoured whereas other molecules were disfavoured and unaffected on solvation. Based on the result of adsorption energy (Table 4) between ions and phytoconstituent showed baicalin and $\mathrm{Ag}$ ion $(-39.58290904 \mathrm{kcal} / \mathrm{mol})$ for the most favourable and stable conformation in the orientation space. The higher energy between baicalin and nitrate ion showed unfavourable binding.

Blends-binding energy calculation of specific complex concluded that the baicalin and Ag ion complex found that more negative energy of $-0.30605 \mathrm{kcal} / \mathrm{mol}$, which indicated that the complex was more stable compared to nitrate ion complex as well as other phytoconstituent complexes. The energy distribution graph revealed that the energy of Ag ion with complex less energy compared with the nitrate and water molecules.

Interaction analysis of AgNPs and phytoconstituent complex inside the amorphous periodic boundary. Especially, 2 cyclic rings of the baicalin form the $\pi$-cationic interaction with four $\mathrm{Ag}$ ions distance range from 3.09-4.01 $\AA$ between the centre of the benzene ring. These AgNPs built the repulsive network, between each other, which shielded the baicalin molecule from the nitrate ion attack (fig. 4). Also, the negative HOMO orbitals of the $\mathrm{Ag}$ ion form interaction with the positive LUMO orbital of baicalin (fig. 3). Based on this information the hypothetical reduction reaction of $\mathrm{AgNO}_{3}$ to AgNPs by the phytoconstituents schemed. The T. procumbens phytoconstituent, baicalin, a flavone glycoside, contained multiple -OH groups and a ketone group accepted an electron to form an interaction between $\mathrm{Ag}^{+}$ions present in the $\mathrm{AgNO}_{3}$ solution. In this probable reaction, baicalin $(-\mathrm{OH} / \mathrm{C}=\mathrm{O})$ hydroxyl group or carboxyl groups form a complex with $\mathrm{Ag}$ ions and $\pi$ cationic interaction with the aromatic rings, which caused the reduction of $\mathrm{Ag}$ ion and stabilization of AgNPs.

The result of the present correlation study between the computational simulation and experimental evaluation concluded that among the 18 phytoconstituents found in the methanol fraction of $T$. procumbens, only baicalin acted as a reducing as well as a stabilizing agent to shape the AgNPs. DFT analysis and blends binding energy clearly explain the HOMO and LUMO orbital interaction as well as the interaction between the adsorbent atoms of baicalin. This tactic would give greater impetus to the growing green synthetic nanoengineering field.

\section{Acknowledgements:}

The authors would like to thank Prof. R. Babu Rajendran, Bharathidasan University, India for the GC-MS facility provided, the Crescent Institute of Science and Technology for running a plagiarism check and Mr. S. Soosairaj, Assistant Professor, Department of Botany, St. Joseph's College, Tiruchirappalli, Tamilnadu, India, for identifying the plant material.

\section{REFERENCES}

1. Zhou K, Wang X, Sun X, Peng Q, Li Y. Enhanced catalytic activity of ceria nanorods from well-defined reactive crystal planes. J Catal 2005;229(1):206-12.

2. Porter LA. Chemical Nanotechnology: A Liberal Arts Approach to a Basic Course in Emerging Interdisciplinary Science and Technology. J Chem Educ 2007;84(2):259.

3. Khan I, Saeed K, Khan I. Nanoparticles: Properties, applications and toxicities. Arab J Chem 2017;05:11.

4. Rai M, Posten C. Green biosynthesis of nanoparticles: mechanisms and applications. Wallingford, Oxfordshire, England: CABI Publishers; 2013.

5. Anjum S, Haider Abbasi B, Khan Shinwari Z. Plant-mediated green synthesis of silver nanoparticles for biomedical applications: challenges and opportunities. Pak J Bot 2016:48(4):1731-60.

6. Cauerhff A, Castro GR. Bionanoparticles, a green nanochemistry approach. Electron J Biotechnol 2013;16(3):110 .

7. Mohanpuria P, Rana NK, Yadav SK. Biosynthesis of nanoparticles: Technological concepts and future applications. J Nanoparticle Res 2008;10(3):507-17.

8. Kole C, Kumar DS, Khodakovskaya MV. Plant Nanotechnology: Principles and Practices. Switzerland: Springer International Publishing; 2018.

9. Metz KM, Sanders SE, Pender JP, Dix MR, Hinds DT, Quinn SJ, et al. Green Synthesis of Metal Nanoparticles via Natural Extracts: The Biogenic Nanoparticle Corona and Its Effects on Reactivity. ACS Sustain Chem Eng 2015;3(7):1610-7.

10. Marchiol L, Mattiello A, Pošćić F, Giordano C, Musetti R. In vivo synthesis of nanomaterials in plants: Location of silver nanoparticles and plant metabolism. Nanoscale Res Lett 2014;9(1):101.

11. Gardea-Torresdey JL, Parsons JG, Gomez E, Peralta-Videa J, Troiani HE, Santiago P, et al. Formation and Growth of $\mathrm{Au}$ Nanoparticles inside Live Alfalfa Plants. Nano Lett 2002;2(4):397.

12. Mohamad NAN, Arham NA, Jai J, Hadi A. Plant Extract as Reducing Agent in Synthesis of Metallic Nanoparticles: A Review. Adv Mater Res 2013;832:350-5.

13. Ikewuchi CC, Ikewuchi JC, Ifeanacho MO. Phytochemical composition of Tridax procumbens Linn leaves : potential as a functional food. Food Nutr Sci 2015;6:992-1004.

14. Ahmad Mir S, Jan Z, Mir S, Dar AM, Chitale G. A Concise 
Review on Biological Activity of Tridax procumbens Linn. Org Chem Curr Res 2017;06(01):1-4.

15. Kethamakka SRP, Deogade MS. Review Article Jayanti veda (Tridax procumbens) - Unnoticed Medicinal plant by Ayurveda. J Indian Sys Med 2014;2(1):6-20.

16. Raveendran S, Sen A, Maekawa T, Kumar DS. Ultra-fast microwave aided synthesis of gold nanocages and structural maneuver studies. Nano Res 2017;10(3):1078-91.

17. Irfan N, Puratchikody A. Advancement of Total Phytoconstituent Extraction Process By New Way Factor Analysis for Tridax procumbens L. Int Res J Pharm 2017;8(10):167-73.

18. Venkat Kumar S, Rajeshkumar S. Plant-Based Synthesis of Nanoparticles and Their Impact. In: Nanomaterials in Plants, Algae, and Microorganisms. Cambridge, Massachusetts, United States: Academic Press; 2018. p. 33-57.

19. Luo K, Jung S, Park KH, Kim YR. Microbial Biosynthesis of Silver Nanoparticles in Different Culture Media. J Agric Food Chem 2018;66(4):957-62.

20. Gurushantha K, Anantharaju KS, Renuka L, Sharma SC, Nagaswarupa HP, Prashantha SC, et al. New green synthesized reduced graphene oxide- $\mathrm{ZrO} 2$ composite as high performance photocatalyst under sunlight. RSC Adv 2017;7(21):12690703.

21. Hou WC, Stuart B, Howes R, Zepp RG. Sunlight-driven reduction of silver ions by natural organic matter: Formation and transformation of silver nanoparticles. Environ Sci Technol 2013;47(14):7713-21.

22. Connors KA. Chemical Kinetics: The Study of Reaction Rates in Chemistry. Hoboken, New Jersey, United States: Wiley; 1990. p. 2, 3, 66.

23. Clogston JD, Patri AK. Zeta Potential Measurement. Methods Mol Biol 2011;697:63-70.

24. Del Bello E, Taddeucci J, De'Michieli Vitturi M, Scarlato P, Andronico D, Scollo S, et al. Effect of particle volume fraction on the settling velocity of volcanic ash particles: Insights from joint experimental and numerical simulations. Sci Rep 2017;7(1):39620.

25. Schultze-Rhonhof E. Die Kristallstruktur des Silbersulfidorthosilicates, Ag8 S2 Si O4. Acta Cryst B 1974;30:2553-8.

26. Hospital A, Goñi JR, Orozco M, Gelpí JL. Molecular dynamics simulations: advances and applications. Adv Appl Bioinform Chem 2015;8:37-47.

27. Khan MAM, Kumar S, Ahamed M, Alrokayan SA, AlSalhi MS. Structural and thermal studies of silver nanoparticles and electrical transport study of their thin films. Nanoscale Res Lett 2011;6:1-8.

28. Monti S, Barcaro G, Sementa L, Carravetta V, Ågren H. Dynamics and self-assembly of bio-functionalized gold nanoparticles in solution: Reactive molecular dynamics simulations. Nano Res 2018;11(4):1757-67.

29. Khazraji AC, Robert S. Interaction effects between cellulose and water in nanocrystalline and amorphous regions: A novel approach using molecular modeling. J Nanomater 2013;2013:409676.

30. Ruiz-Morales Y. HOMO-LUMO gap as an index of molecular size and structure for polycyclic aromatic hydrocarbons (PAHs) and asphaltenes: A theoretical study. I. J Phys Chem A 2002;106(46):11283-308.

31. Jain S, Mehata MS. Medicinal Plant Leaf Extract and Pure Flavonoid Mediated Green Synthesis of Silver Nanoparticles and their Enhanced Antibacterial Property. Sci Rep 2017;7(1):1-13.

32. Mendieta-Moreno JI, Marcos-Alcalde I, Trabada DG, Gómez-Puertas P, Ortega J, Mendieta J. A practical quantum mechanics molecular mechanics method for the dynamical study of reactions in biomolecules. Adv Protein Chem Struct Biol 2015;100:67-88.

33. Fennell CJ, Dill KA. Physical Modeling of Aqueous Solvation. J Stat Phys 2011;145:209-26.

34. Suzuki T, Okita M, Kakoyama S, Niinae M, Nakata H, Fujii $\mathrm{H}$, et al. Preferential adsorption and surface precipitation of lead(II) ions onto anatase in artificially contaminated Dixie clay. J Hazard Mater 2017;338:482-90.

35. Ting ECM, Popa T, Paci I. Surface-site reactivity in smallmolecule adsorption: A theoretical study of thiol binding on multi-coordinated gold clusters. Beilstein J Nanotechnol 2016;7(1):53-61.

36. Zhang W, Gomez ED, Milner ST. Predicting Flory-Huggins $\chi$ from Simulations. Phys Rev Lett 2017;119(1):017801.

37. Akkermans RLC, Spenley NA, Robertson SH. Monte carlo methods in materials studio. Mol Simul 2013;39(14-15):115364.

38. Noguez C. Surface plasmons on metal nanoparticles: The influence of shape and physical environment. J Phys Chem C 2007;111(10):3606-19.

39. Chorsi HT, Zhu Y, Zhang JXJ. Patterned Plasmonic SurfacesTheory, Fabrication, and Applications in Biosensing. J Microelectromech Syst 2017;26(4):718-39.

40. Lee KS, El-Sayed MA. Gold and silver nanoparticles in sensing and imaging: Sensitivity of plasmon response to size, shape, and metal composition. J Phys Chem B 2006;110(39):19220-5.

41. Bogireddy NKR, Pal U, Gomez LM, Agarwal V. Size controlled green synthesis of gold nanoparticles using Coffea arabica seed extract and their catalytic performance in 4-nitrophenol reduction. RSC Adv 2018;8(44):24819-26.

42. Shah M, Fawcett D, Sharma S, Tripathy S, Poinern G, Shah M, et al. Green Synthesis of Metallic Nanoparticles via Biological Entities. Materials 2015;8(11):7278-308.

43. Liu A, Ren X, An M, Zhang J, Yang P, Wang B, et al. A Combined Theoretical and Experimental Study for Silver Electroplating. Sci Rep 2015;4(1):3837.

44. Otero R, Vázquez de Parga AL, Gallego JM. Electronic, structural and chemical effects of charge-transfer at organic/ inorganic interfaces. Surface Sci Rep 2017;72:105-45. 